\title{
Active Fibre Composites: Optimization of the manufacturing process and their poling behavior
}

\author{
Christian Huber", Doris M. Spori, Mark M. Melnykowycz, Michel Barbezat \\ Empa - Materials Science \& Technology, \\ Ueberlandstr. 129, CH-8600 Dübendorf, Switzerland
}

\begin{abstract}
The scientific community has put significant efforts into the manufacturing and optimization of sensors and actuators made of piezoelectric fibres with interdigitated electrodes, well known as Active Fibre Composites (AFC). A great advantage of such AFC is their flexibility and the possibility to integrate them into composite structures.

In the current study an approach of optimizing the manufacturing process as well as the polarization of AFCs utilizing piezoelectric Lead-Zirconate-Titanate (PZT) fibres embedded in an epoxy matrix between interdigital Electrodes (IDE) screenprinted on Kapton will be discussed. During the poling process, an electric field is applied over the interdigitated electrodes of the AFC to its piezoelectric fibres along the fibre axis. One of the most important parameters of this polarization is - beside temperature and time - the applied voltage. An increase of the electric field results in an increase of the AFCs performance as shown by free-strain measurements.

The manufacturing process developed and used at Empa consists of laminating the piezoelectric fibres in an epoxy. matrix between the electrodes. An essential goal of this lamination - carried out in a hot press - is to get a proper contact between piezo fibres and the electrode. By adding soft layers between the Kapton foil and the mould, the interdigitated electrodes are deformed by each single fibre and therefore build up a contact area which in its cross section can be described by a contact angle. This optimization of the manufacturing process is also shown by free strain measurements of the AFC.
\end{abstract}

Keywords: Active Fibre Composites, PZT, Characterization, Optimization

\section{INTRODUCTION}

Active Fibre Composites (AFC) are constituted of uniaxially aligned piezoceramic fibres (PZT) [1] embedded in a polymer matrix and sandwiched between two sets of interdigitated electrodes. When an electric voltage is applied between the interdigitated electrodes, the electric field generated in the fibres results in an extension of the AFC in the direction parallel to the fibre axis (see sketch of AFC in Figure 1). Inversely, the PZT-fibres generate electric charges upon mechanical deformation. Therefore, piezoelectric AFC elements can be used as both, actuators and sensors in smart composites and structures. Applications are envisaged, e.g., in structural health monitoring, active structural control, and passive or active vibration damping [2]. Major advantages of the AFC over conventional piezoelectric elements are their flexibility and toughness, both far superior compared with monolithic PZT-wafers. The piezoelectric properties of the PZT-based AFC, on the other hand, are better than those of piezopolymers, such as Polyvinylidene fluoride (PVDF). Furthermore, due to their thin, planar geometry the AFC can be easily integrated into composite laminates [3].

The present study aims at optimisation of the AFC manufacturing process used at Empa, involving lamination of commercial PZT-fibres. AFC elements are characterised at various stages of the manufacturing process.

The pressure applied during curing allows for deformation and adaptation of the electrodes, made from silver paste screen printed on polyimide films, to the shape of the individual fibres [4]. This yields an area of full contact and a contact angle between fibre and electrode of up to $60^{\circ}-70^{\circ}$. The larger the value of the contact angle, the higher the electric field that develops in the direction of the fibre axis upon application of the voltage. An other aspect in the field of contacting the piezoelectric fibre is their variance in diameter. In fact, a hard mould makes difficult to build up a contact area for every single fibre particularly when a fibre with small diameter is located next to a larger one. Therefore a series of soft layers were added in the lamination process what allows to contact each single fibre over a comparable

\footnotetext{
* Corresponding author. Tel.: +41-44-823-4463; fax: +41-44-823-4496. e-mail: christian.huber@empa.ch
} 
contact area. This significantly affects the AFC performance, e.g. as shown by the free strain behaviour measured by strain gauges applied on both sides of the AFC.

The polarisation of the PZT-fibres of the AFC is performed via the interdigitated electrodes. These yields axially poled fibres and thus coincides with the $\mathrm{d}_{33}$ piezoelectric charge coefficient. Electric polarisation of the fibres is characterised by temperature, voltage and duration. In this study the voltage was optimised for maximum actuation of an $\mathrm{AFC}$ as measured by the strain gauges.

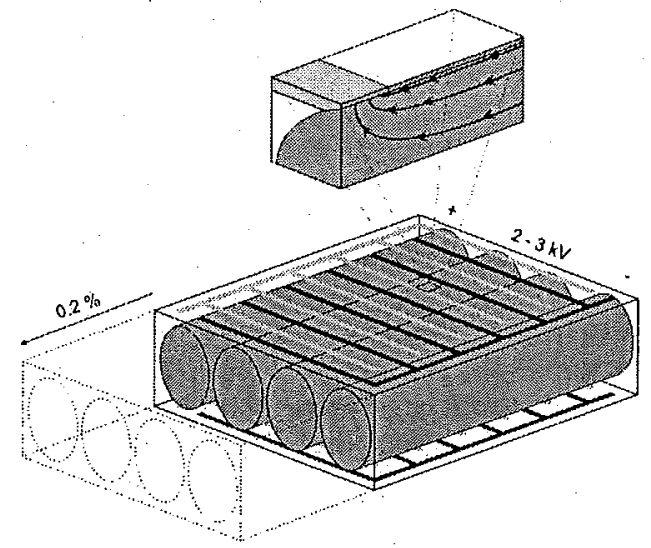

Figure 1: Sketch of an active fibre composite.

\section{MATERIAL \& METHODES}

\subsection{Materials}

The investigated AFCs consist of piezoelectric fibres (PZT-5A) with a diameter of $264 \mu \mathrm{m}( \pm 5 \mu \mathrm{m})$. Polyimide foils (Kapton $100 \mathrm{HN} 25 \mu \mathrm{m}$ in thickness) were used for the preparation of the screen-printed interdigitated electrodes with a silver filled epoxy paste. A low viscosity two-component epoxy resin with an antifoaming agent was used as matrix for the Active Fibre Composite. Isolated copper wires and a silver paste allow contacting the electrodes to the high voltage source.

\subsection{AFC Manufacture}

Active Fibre Composites were manufactured according to a similar procedure than the one described earlier by Bent [5]. A fibre mat was first prepared with the piezoelectric fibres in order to orient them parallel to one another. The fibre mat was then transferred between the interdigitated electrodes and the epoxy matrix was added. The active fibre composite was contacted with cables and placed under a hydraulic press at $120^{\circ} \mathrm{C}$. High pressure (up to $3.4 \mathrm{MPa}$ ) was used in order to optimize the contact between the fibres and the interdigitated electrodes. The standard AFC was laminated between aluminum plates. For the optimization of the manufacturing process, the lamination was carried out under the same conditions in the hot press with added soft layers between AFC and aluminum plates. These soft layers consist of a silicone sheet $(2.0 \mathrm{~mm}$ in thickness, 70 ShoreA), Teflon foil $(0.315 \mathrm{~mm}$ in thickness) or cardboard sheet $(1.34 \mathrm{~mm}$ in thickness).

The AFC was cured for 1 hour at $120^{\circ} \mathrm{C}$ and post cured 8 hours at $160^{\circ} \mathrm{C}$. All AFCs were polarized with an electrical voltage of $2.78 \mathrm{kV} / \mathrm{mm}$ (the applied electric field is indicated in volts per mm electrode spacing) in an oven at a temperature of $80^{\circ} \mathrm{C}$ for 20 minutes. For the polarization experiments, the same AFC was poled in steps of $0.5 \mathrm{kV} / \mathrm{mm}$ with characterization measurements in between.

The dimensions of the AFCs were: $31 \mathrm{~mm}$ in length, $20 \mathrm{~mm}$ in width (active area) and approximately $310 \mu \mathrm{m}$ in thickness. The electrode finger width was $200 \mu \mathrm{m}$ and the finger spacing was $0.9 \mathrm{~mm}$.

\subsection{AFC Characterization}

The direct free strain measurements were obtained using standard electrical resistance foil strain gauges $(10 \mathrm{~mm}$ in length) bonded symmetrically on both sides of the AFC and connected in series to an amplifier. Electric fields between - 
1 and $3 \mathrm{kV} / \mathrm{mm}$ were applied to the AFCs in order to generate characteristic activation curves (i.e. work cycles). For the poling study, the applied electric field was between 0 and the applied poling voltage.

\section{RESULTS \& DISCUSSION}

The free strain curves presented in Figure 2 a-d were obtained by applying an electric field between -1 and $3 \mathrm{kV} / \mathrm{mm}$. These work cycles were measured with strain gauges on four AFCs with different laminating process. Compared to the standard type AFC (figure 2 a) which is laminated in a hot press between two aluminium plates, the other three AFCs were laminated with some additional soft layers between the AFC laminate and the same aluminium mould of the standard type processing. These soft layers consist of cardboard, silicone film as well as a Teflon film.

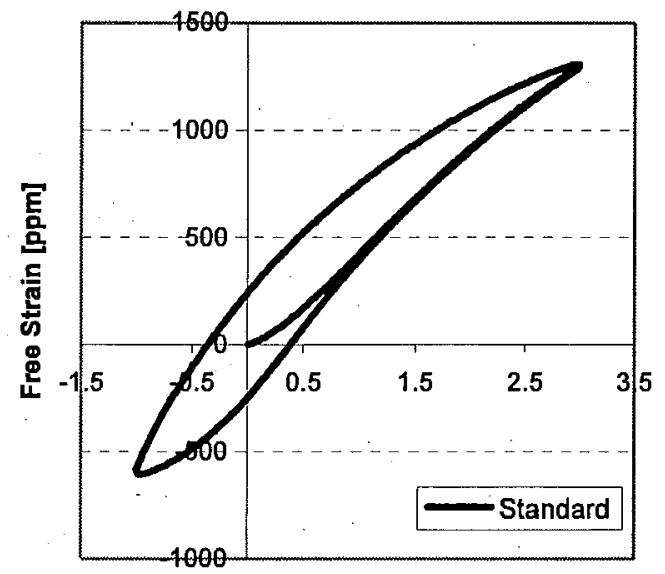

Applied Voltage [kV/mm]

$\mathbf{A}$

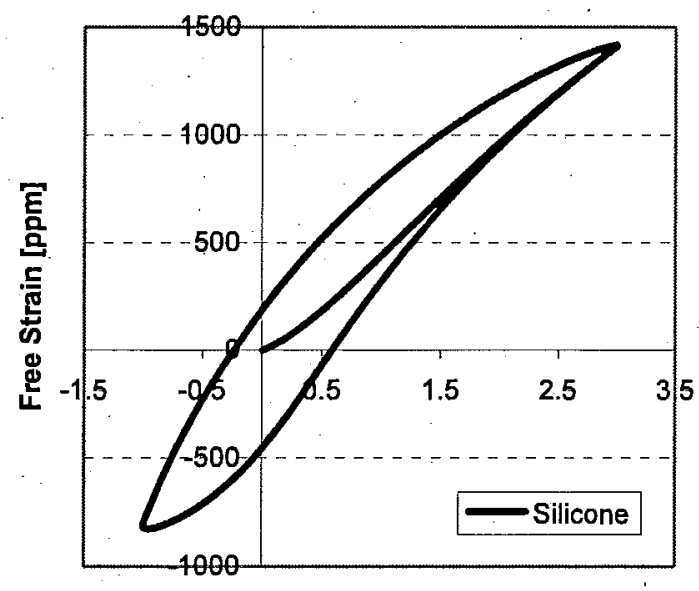

Applied Voltage $[\mathrm{kV} / \mathrm{mm}]$

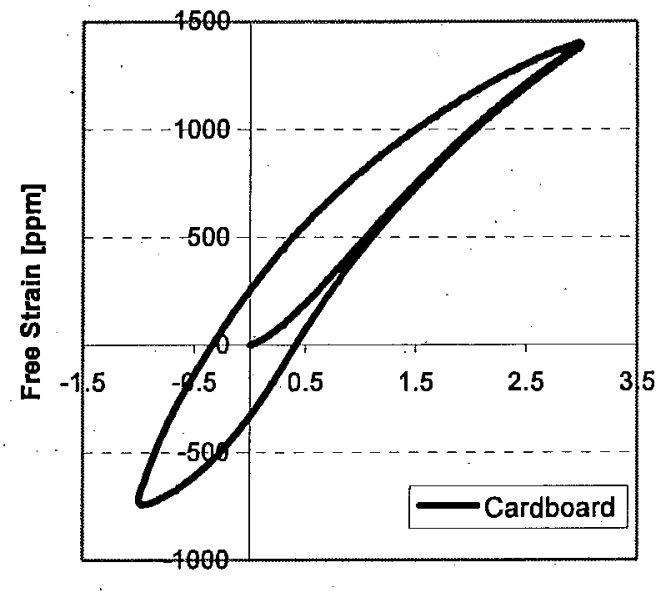

Applied Voltage $[\mathbf{k V} / \mathrm{mm}]$

B

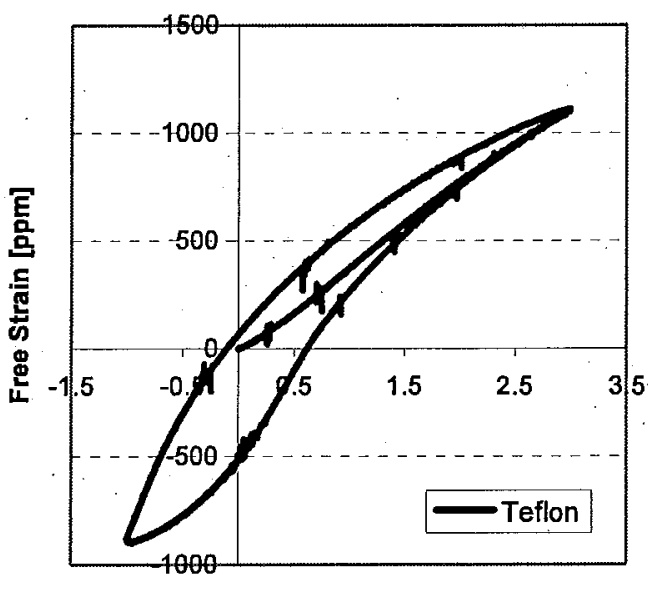

Applied Voltage [kV/mm]

C

d

Figure 2: Workcycles of AFCs with different interlayer. Standard type AFC laminated between aluminium plates (a), AFCs laminated with cardboard (b), silicone (c) and Teflon (d) interlayer. 
The most obvious change in the AFC-performance due to the soft interlayer can be seen in Fig 2 a-d in the free strain where an increase of more than $17 \%$ is detectable compared to the standard type AFC. Table 1 shows the measured values of the free strain, the $d_{33}$ at high $(1.8-3 \mathrm{kV} / \mathrm{mm})$ as well as at low field $(0-0.2 \mathrm{kV} / \mathrm{mm})$, the capacity and the AFC-thickness. These properties show an increase of the AFC-performance by laminating between soft layers that can be explained by a better contacting between the piezoelectric fibres and the interdigitated electrodes. Other studies showed an increase of the AFC performances by applying higher pressure during the lamination to get larger contact area between the finger electrodes and the PZT fibres. In fact, this increase of pressure results in larger contact angle which was measured on an AFC cross section between fibre and electrode - as well as in lower thicknesses what can be explained by a deformation. of the Kapton foil and the electrode [6]. By laminating the AFCs with soft layers, the Kapton foil will not be compressed, resulting in higher thicknesses compared to the standard type AFC. The increase of the performance by laminating the AFC between soft layers can be explained by a better contacting of each single fibre. Due to a relatively wide range of fibre cross section dimension the contacting of a fibre with smaller diameter lying next to one with large diameter is difficult when laminated with a hard aluminium mould. The soft layers allowed a proper contacting also of these fibres and a contacting without deforming the Kapton foil in its thickness.

\begin{tabular}{lccccc}
\hline & Aluminium & Teflon & Cardboard & Silicone \\
\hline Free Strain $[\mathrm{ppm}]$ & $1915.53 \pm 4.93$ & $2012.24 \pm 13.77$ & $2136.85 \pm 5.27$ & $2248.76 \pm 3.07$ \\
d 33 High Field $[\mathrm{pm} / \mathrm{V}]$ & $387.79 \pm 12.21$ & $355.97 \pm 11.55$ & $399.30 \pm 11.98$ & $458.70 \pm 16.00$ \\
$\mathrm{~d} 33$ Low Field $[\mathrm{pm} / \mathrm{V}]$ & $253.20 \pm 9.60$ & $272.11 \pm 7.67$ & $282.90 \pm 8.99$ & $285.49 \pm 3.28$ \\
Capacitance $[\mathrm{pF}]$ & 787.2 & 976.1 & 1009 & 864.2 \\
Thickness $[\mu \mathrm{m}]$ & $321 \pm 9.71$ & $336 \pm 8.32$ & $337 \pm 11.31$ & $331 \pm 6.50$ \\
\hline
\end{tabular}

Table 1: Values of free strain, $d_{33}$ at high field and low field, capacitance and thickness taken from AFC work cycles of standard type AFC laminated between aluminium plates and AFCs laminated with Teflon, cardboard and silicone interlayer.

An other aspect of this study is the optimization of the AFC polarisation process - in fact the polarisation voltage. The polarisation of $\mathrm{AFC}$ is carried out by applying an electric field through the piezoelectric fibres by applying a voltage to the interdigitated electrodes for a certain time at elevated temperatures. Other studies [7] showed an optimum poling temperature at $80^{\circ} \mathrm{C}$ with an applied electric field for twenty minutes. This study deals with the applied voltage during this poling process. Therefore, an AFC was poled for 20 minutes at $80^{\circ} \mathrm{C}$ at a voltage of $0.56 \mathrm{kV} / \mathrm{mm}$. After poling, the free strain was measured with strain gauges from 0 to $0.5 \mathrm{kV} / \mathrm{mm}$. A second poling was carried out at a voltage of 1 $\mathrm{kV} / \mathrm{mm}$, again with a measurement of the free strain form 0 to $0.5 \mathrm{kV} / \mathrm{mm}$ as well as from 0 to $1 \mathrm{kV} / \mathrm{mm}$. This procedure was iterated with poling voltages of $1.5 \mathrm{kV} / \mathrm{mm}$ and $2 \mathrm{kV} / \mathrm{mm}$ and with additional free strain measurements from 0 to $1.5 \mathrm{kV} / \mathrm{mm}$ and from 0 to $2 \mathrm{kV} / \mathrm{mm}$. Figure 3 shows the free strain at loads from 0 to $0.56,1,1.5$ and 2 $\mathrm{kV} / \mathrm{mm}$ for the different poling voltages. It is obvious, that poling below the coercive field - which is for the used PZT fibres around $1 \mathrm{kV} / \mathrm{mm}$ - results in almost none free strain. Above this field -1.5 and $2 \mathrm{kV} / \mathrm{mm}-$ the free strain increases clearly; but the change in the AFCs response is negligible for higher poling voltage if measured at low field. Therefore, it will be useful to polarize the AFC with a voltage which is above the coercive field, what allows an orientation of the piezoelectric dipoles along the applied electric field, but just as high as the voltage for the application will be. 


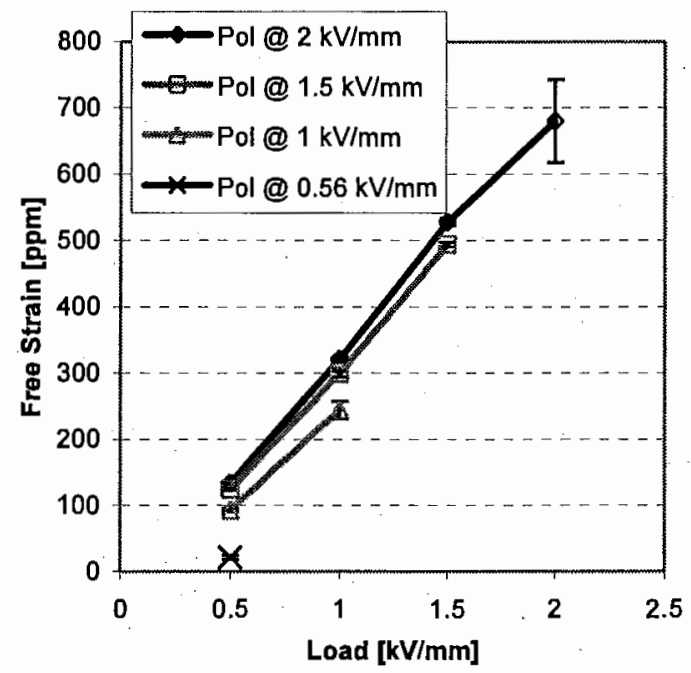

Figure 3: Free strain at different loads from 0 to 0.5 up to 0 to $2 \mathrm{kV} / \mathrm{mm}$ from AFC polarised at different polarization voltages.

The results of this polarization voltage optimization are summarised in table 2.

\begin{tabular}{lcccc}
\hline Polingvoltage $[\mathrm{kV} / \mathrm{mm}]$ & $\mathbf{0 . 5 6}$ & $\mathbf{1 . 0 0}$ & $\mathbf{1 . 5 0}$ & $\mathbf{2 . 0 0}$ \\
\hline Free Strain 0 - 0.5 kV/mm [ppm] & $21.58 \pm 2.88$ & $93.43 \pm 2.68$ & $124.87 \pm 1.38$ & $134.04 \pm 0.87$ \\
Free Strain 0 - $1.0 \mathrm{kV} / \mathrm{mm}[\mathrm{ppm}]$ & - & $245.08 \pm 13.08$ & $299.12 \pm 4.43$ & $320.82 \pm 3.21$ \\
Free Strain 0 - $1.5 \mathrm{kV} / \mathrm{mm}[\mathrm{ppm}]$ & - & - & $494.08 \pm 3.56$ & $527.82 \pm 4.83$ \\
Free Strain 0 - $2.0 \mathrm{kV} / \mathrm{mm}[\mathrm{ppm}]$ & - & - & - & $679.87 \pm 62.7$ \\
\hline
\end{tabular}

Table 2: Values of free strain at different loads for an $\mathrm{AFC}$ polarized at $0.56,1.0,1.5$ and $2.0 \mathrm{kV} / \mathrm{mm}$.

\section{CONCLUSION}

A common way to improve the AFC performance is to enlarge the contact area between the piezoelectric PZT fibre and the screenprinted interdigitated electrode. An alternative possibility to the increase of the pressure during the lamination process with hard aluminium plates, and therefore a compression of kapton foil and electrode around the fibre, is the use of soft layers between the hard aluminium mould and the AFC laminate due to a proper distribution of the applied pressure over every single fibre. One aim of this work was to show the enhancement of the AFC performance by using such soft layers in form of cardboard, silicone film as well as Teflon film and the comparison of AFC produced in this way.

A second goal of the present study was to show the influence of the polarisation on the AFC performance. We were able to show on standard type AFC that it is necessary to raise the polarisation voltage above the coercive filed of the piezoelectric PZT material to get any polarisation. In fact, the higher the poling voltage, the better the performance of the AFC will be, but the gain of free strain will become smaller at higher poling voltages. Additionally the risk of electrical shorts rises with higher applied voltages. Therefore, it will be useful to polarize the AFC with a voltage which is above the coercive field but just as high as the voltage for the application will be. 


\section{ACKNOWLEDGMENTS}

Support for this research has been provided by a grant from Empa Duebendorf.

The technical support of Mr L. DeBoni of the Polymer/Composites Laboratory at Empa for the manufacture of the AFCs is gratefully acknowledged.

\section{REFERENCES}

[1] X. Kornmann, C. Huber, J. Europ. Ceram. Soc., 24 (2004), 1987-1991.

[2] M. Barbezat, A.J. Brunner, P. Flüeler, C. Huber, X. Kornmann, Sensors and Actuators A, 114 (2004), $13-20$.

[3] L.J. Nelson, Material Science and Technology, 18 (2002), 1245-1256.

[4] W.K. Wilkie, R.G. Bryant, J.W. High, R.L. Fox, R.F. Hellbaum, A. Jalink, B.D. Little, P.H. Mirick, Proc. SPIE, 3991 (2000), 323-334.

[5] A. Bent, N.W. Hagood, J. Intell. Mater. Syst. Struct., 8 (11) (1997), 903-919.

[6] A. Belloli, B. Castelli, X. Kornmann, C. Huber, P. Ermanni, Proc. SPIE, 5390 (2004), 78-88.

[7] C. Huber, M. Barbezat, A.J. Brunner, X. Kornmann, Proc. ACTUATOR 2004, 9 (2004), 435-437. 